УДК 539.216:539.213:539.264

DOI: https://doi.org/10.17308/kcmf.2019.21/760

Поступила в редакцию11.03.2019

Подписана в печать 15.05.2019

\title{
ОПЫТ КУЛОНОМЕТРИЧЕСКОЙ ОЦЕНКИ КОРРОЗИОННОАКТИВНОЙ ПОВЕРХНОСТИ АУСТЕНИТНОЙ СТАЛИ В АГРЕССИВНОМ ЭЛЕКТРОЛИТЕ ПРИ ЗНАКОПЕРЕМЕННОЙ ДЕФОРМАЦИИ
}

\author{
( 2019 А. Е. Кузмак $\bowtie$, Н. Е. Есипова, А. В. Кожеуров \\ Институт физической химии и электрохимии им. А. Н. Фрумкина РАН, \\ Ленинский пр. 31, 119071 Москва, Российская Федерация
}

\begin{abstract}
Аннотация. Исследовано коррозионное поведение сложнолегированного сплава в растворе 3M HCl при статической деформации переменного знака. Результаты, полученные методом кулонометрической регистрации продуктов коррозии в начальный период взаимодействия металла со средой, обеспечили количественную оценку специфики растворения сплава. Полученные результаты позволили объемным методом в сочетании с методом кулонометрической регистрации продуктов коррозии разработать методологию селективной оценки коррозионного поведения многофазных сплавов в условиях агрессивной среды.
\end{abstract}

Ключевые слова: коррозия, деформация, кулонометрия, волюмометрия, поверхностное напряжение.

\section{ВВЕДЕНИЕ}

Многочисленные механохимические эксперименты с твердым телом, в том числе и коррозионные испытания напряженных стальных образцов простого и сложного состава в упругой области показали разную скорость растворения в сжатой и растянутой зонах [1-5]. Это явление ранее было теоретически предсказано и получило название механохимического эффекта знака деформации [6]. Авторы использовали уравнения теории упругости для оценки влияния вида деформирования на коррозию металла в кислом электролите. Объяснение причины этой асимметрии заключается в наличии поверхностного напряжения, самопроизвольно образующегося из-за поверхностного натяжения, присутствующего во всех телах. Если в начальном состоянии на поверхности уже имеется натяжение, то при растяжении поверхности оно складывается с приложенным напряжением, а при сжатии вычитается из него. Симметрия сторон исчезает, и скорость любого физико-химического процесса зависит от знака деформации. Предсказанный эффект для процесса коррозии (растворения) особенно важен, так как коррозия - явление поверхностное.

$\square$ Кузмак Александр Евсеевич, e-mail: akuzmak@yandex.ru
Механохимический эксперимент по исследованию коррозионной кинетики обычно проводился объёмным (водородным) методом в кислом электролите [7]. Однако при использовании объёмного метода обычно регистрируют кинетику интегрального накопления деполяризатора (водорода или кислорода) при параллельном растворении всех компонентов сплава, исключая, тем самым, корректную регистрацию коррозионной кинетики в начальный период коррозии. С физической точки зрения при одновременном воздействии механического деформирования и агрессивной среды в реакционном пространстве «твёрдое тело-электролит» возникают колебательно - и электронно-возбужденные состояния межатомных связей (в т. ч. свободные радикалы, координационно-ненасыщенные атомы, структурные дефекты, заряженные центры), стимулирующие коррозию компонентов сплава и, как следствие, формирование слоя продуктов взаимодействия металла со средой. Нестабильный по структуре, упомянутый слой изменяет толщину в процессе формирования, что вносит неопределённость в результаты исследования коррозионной кинетики традиционными методами в начальный период коррозионного процесса. Исследование состава 
оксидного слоя, изучение кинетики пассивации и депассивации представляет собой самостоятельную задачу.

Проведенные коррозионные испытания многофункциональных сплавов привели к развитию и постановке новых задач. Как показано в работах [8-10], эффективным подходом к решению проблемы количественной регистрации микроколичеств вещества для систем с различным структурно-фазовым состоянием поверхности служит метод кулонометрической регистрации продуктов коррозии (метод КРПК). Сочетание и сопоставление возможностей методов электрохимического ионного анализа КРПК с интегральным (объёмным) для разработки методологии оценки влияния знака деформации на коррозионную кинетику многофазного сплава стало целью настоящей работы.

В работе исследовали кинетику растворения сложнолегированной аустенитной стали марки 05Х22АГ15Н8М2Ф-Ш (железо 55 \%, хром 20 \%, никель 8 \%, марганец $15 \%$, азот $0.5 \%$, сера < 0.005) под напряжением в упругой области. Данный сплав обладает высоким сопротивлением к динамической нагрузке, способностью к деформационному упрочнению и коррозионной стойкостью, обеспеченную направленным легированием [8]. Поскольку этот вид стали находит широкое применение в медицине, судостроении, при производстве химического оборудования и в других сферах, изучение влияния вида деформации (растяжение-сжатие) на коррозионную кинетику в агрессивном электролите представляет практический интерес.

\section{ЭКСПЕРИМЕНТАЛЬНАЯ ЧАСТЬ}

Опыты проводились на начальном временном участке коррозии с целью недопущения развития микротрещин. Испытательной ячейкой служил химический стакан, на дно которого помещали специальные устройства с испытуемым образцом [5]. Устройство обеспечивало фиксированный радиус кривизны пластинки (сжатой и растянутой поверхности). Стакан заполняли $250 \mathrm{ml} 3 \mathrm{M}$ раствором $\mathrm{HCl}$. Воздействию агрессивной среды подвергали область деформированной пластинки площадью $S=1.5 \times 2.0 \mathrm{~cm}^{2}$. Нерабочую поверхность изолировали химически стойким изоляционным материалом. Испытания проводили без перемешивания среды при комнатной температуре.

Кулонометрический анализ определял содержание ионов $\mathrm{Fe}^{2+}$ и $\mathrm{Fe}^{3+}$ согласно методике [9], начиная со времени экспозиции $t=20 \mathrm{~min}$. Далее, через каждые 20 min и до 140 min испытаний отбирали аликвоты коррозионно-агрессивной среды объемом $8 \mathrm{ml}$, которые помещали в кулонометрическую ячейку-индикатор из стеклоуглерода СУ-2000. Тестировали сжатые и растянутые пластины сплава с радиусами кривизны, соответственно: $R_{\text {сж }}=104.0 ; 44.0 ; 31.8 ; 21.0 \mathrm{~mm}$; $R_{\text {раст }}=97.0 ; 25.0 ; 16.1 ; 10.9$ mm. Для интегральных оценок потерь массы образцов использовали объёмный (водородный) метод [4].

\section{ОБСУЖДЕНИЕ РЕЗУЛЬТАТОВ}

На рис. 1 приведены кинетические зависимости накопления ионов - продуктов коррозии $\mathrm{Fe}^{2+}$ и $\mathrm{Fe}^{3+}$ в среде при различных радиусах кривизны сжатой и растянутой поверхностей стали в условиях стационарного напряжения с использованием метода КРПК. Кривые демонстрируют особенности процесса накопления продуктов коррозии на сжатой поверхности образца. Как видно из рис. 1, на сжатой поверхности отсутствует продукт коррозии в виде $\mathrm{Fe}^{2+}$. В данном случае, очевидно, идёт окисление $\mathrm{Fe}^{2+} \rightarrow \mathrm{Fe}^{3+}$ и удержание $\mathrm{Fe}^{2+}$ в виде оксида на поверхности образца.

По ионам $\mathrm{Fe}^{3+}$, перешедшим в среду, идентифицируют только минимально нагруженный

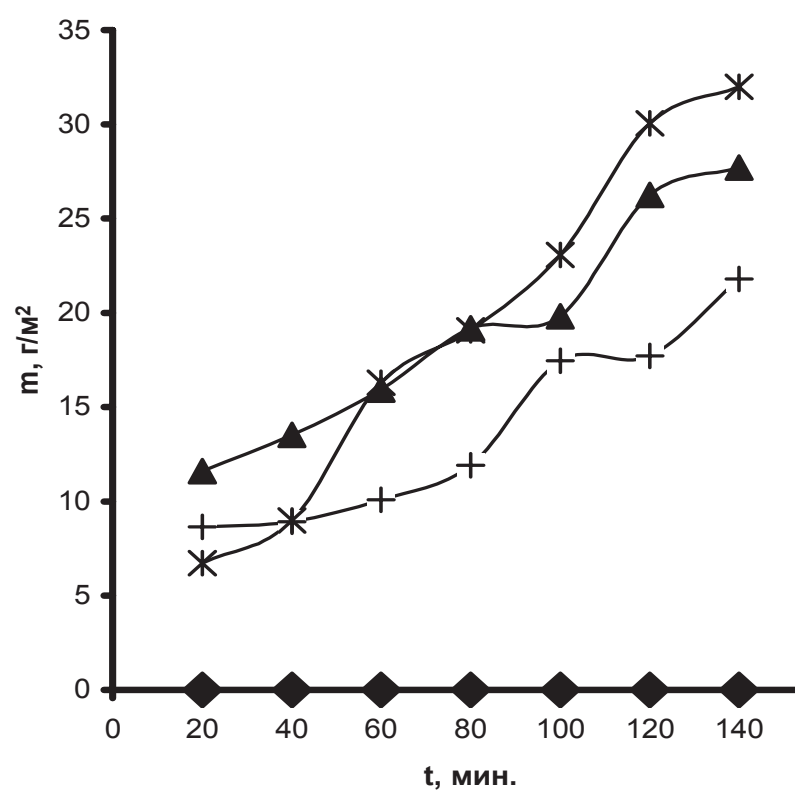

Рис. 1 Кинетика накопления продуктов коррозии на сжатой поверхности. - $\mathrm{Fe}^{2+}$ для всех $R,+-\mathrm{Fe}^{3+}$ для $R=104 \mathrm{Mм}, \boldsymbol{\Delta}-\mathrm{Fe}^{3+}$ для прочих $R, *-\mathrm{Fe}^{3+}$ для плоской поверхности

[Fig. 1 The kinetics of accumulation of corrosion products on a compressed surface. $-\mathrm{Fe}^{2+}$ for all $R$; $+-\mathrm{Fe}^{3+}$ for $R=104 \mathrm{~mm} ; \boldsymbol{\Delta}-\mathrm{Fe}^{3+}$ for other $R ; *-\mathrm{Fe}^{3+}$ for a flat] 
образец с радиусом кривизны $R_{\text {сж }}=104 \mathrm{~mm}(+)$. При увеличении нагрузки (уменьшения радиуса кривизны) выход $\mathrm{Fe}^{3+}$ возрастает в $1.3 \div 1.4$ раза, однако различий в количестве ионов $\mathrm{Fe}^{3+}$ при других радиусах $(\bullet ; \mathbf{\Delta})$ не установлено. При испытаниях ненагруженной пластины, ионов $\mathrm{Fe}^{2+}$ (*) также не обнаружено.

Выход ионов $\mathrm{Fe}^{3+}$ за период до $40 \mathrm{~min}$, примерно, соответствует минимально нагруженному образцу, в интервале от 60 до $80 \mathrm{~min}$ - образцам с радиусами $R_{\text {сж }}=44.0,31.0$, и $21.0 \mathrm{~mm}$, а в интервале от 100 до 140 min превышает показатели последних трех на 15 \%. Учитывая данные, полученные на сжатой и ненагруженной поверхностях (рис. 1), можно предположить, что формирующийся слой обладает в рассматриваемой среде защитным действием.

На растянутой поверхности (рис. 2) ионов $\mathrm{Fe}^{2+}$ не обнаружено в интервале времени от 0 до 80-100 минут.

Далее железо (рис. 3), быстро накапливается в среде, особенно - на максимально нагруженном образце $\left(R_{\text {раст }}=10.9 \mathrm{~mm}\right)$.

При этом прекращается накопление ионов $\mathrm{Fe}^{3+}$, так как в указанный выше период запас кислорода в реакционной зоне полностью израсходован. Происходит растворение слоя продуктов коррозии, а также окисление железа исключительно по реакции $\mathrm{Fe}^{\circ}+2 \mathrm{H}^{+} \rightarrow \mathrm{Fe}^{2+}+2 \mathrm{H}^{\circ}$ (что позволяет идентифицировать нагрузки на образцах).

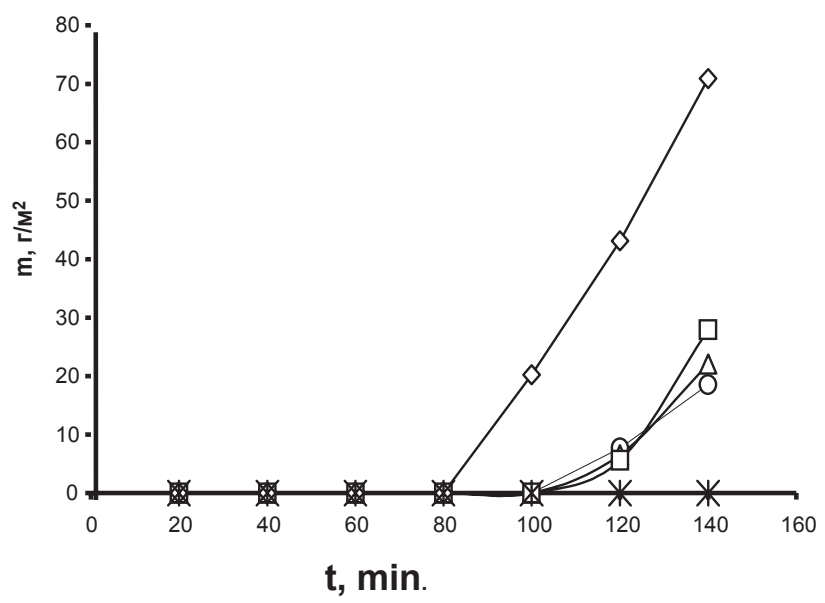

Рис. 2 Кинетика накопления $\mathrm{Fe}^{2+}$ на растянутой поверхности: $R=25$ мм; $\square-R=16.1 \mathrm{мм} ; \diamond-$ $R=10.9$ мм; $\Delta \circ-R=97$ мм; $*$ - плоская поверхность

[Fig. 2. The kinetics of accumulation of $\mathrm{Fe}^{2+}$ on the stretched surface $R=25 \mathrm{~mm}, \square-R=16.1 \mathrm{~mm} ; \diamond-$ $R=10.9 \mathrm{~mm} ; \Delta \circ-R=97 \mathrm{MM} ; *-$ flat surface $]$
В связи с тем, что регистрация количества ионов $\mathrm{Fe}^{2+}$ обеспечивает оценку кинетики в зависимости от радиуса кривизны образца, в настоящей работе проведено сравнение данных, полученных методами КРПК, и расчетных - по количеству выделившегося водорода на поверхностях образцов с различными радиусами кривизны (таблица).

Как видно из таблицы на ненагруженной поверхности количество $\mathrm{Fe}^{2+}$, рассчитанное по водороду, примерно, соответствует железу, определенному методом КРПК, только через $140 \mathrm{~min}$ от начала испытаний. Ранее, кулонометрические показатели превышают водородные, что связано с наличием двух видов деполяризаторов в реакционном объеме: кислорода и иона $\mathrm{H}^{+}$.

Это связано с тем, что при объёмной регистрации фиксируется только количество ионов $\mathrm{Fe}^{2+}$, полученное по реакции $\mathrm{Fe}^{\circ}+2 \mathrm{H}^{+} \rightarrow \mathrm{Fe}^{2+}+2 \mathrm{H}^{\circ}$, а при кулонометрических измерениях к нему добавляется железо, полученное в результате частичного растворения защитного (оксидного) слоя. Процесс убыли кислорода в реакционном пространстве сближает показатели кулонометрической и водородной регистрации.

На сжатой поверхности ситуация аналогична за исключением того, что показатели водородной и кулонометрической регистрации железа сближаются медленнее, свидетельствуя

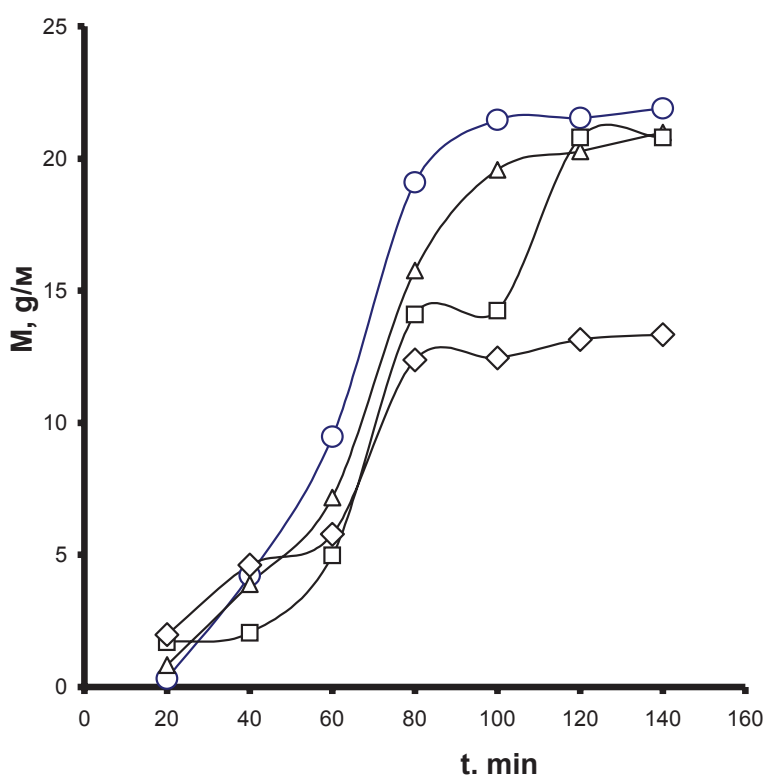

Рис. 3 Кинетика накопления $\mathrm{Fe}^{3+}$ на растянутой поверхности: $\Delta-R=25$ мм, $\square-R=16.1 \mathrm{мm}, \diamond-$ $R=10.9$ мм, $*-$ плоская поверхность (не показана)

[Fig. 3. The kinetics of accumulation of $\mathrm{Fe}^{3+}$ on the stretched surface. $\Delta-R=25 \mathrm{~mm}$; $\square-R=16.1 \mathrm{~mm}$; $\diamond-R=10.9 \mathrm{~mm} ; *-$ flat surface (not shown)] 
Таблица. Накопление в среде ионов $\mathrm{Fe}^{2+}$ и $\mathrm{Fe}^{2+}+\mathrm{Fe}^{3+}$, зарегистрированное водородным и КРПК методами

[Table. Accumulation in the environment of ions $\mathrm{Fe}^{2+}$ and $\mathrm{Fe}^{2+}+\mathrm{Fe}^{3+}$, registered hydrogen and CDCP method]

\begin{tabular}{|c|c|c|c|c|c|c|c|c|c|}
\hline \multirow{3}{*}{$\begin{array}{c}\text { Время } \\
\text { от } \\
\text { начала } \\
\text { испыт. } \\
\text { мин } \\
\text { [Time] }\end{array}$} & \multicolumn{3}{|c|}{$\begin{array}{c}\text { Растянутая поверхность, } \\
\text { Rраст. }=10.9 \mathrm{~mm} \\
\text { [Stretched surface, } R_{\text {str. }}=10.9 \\
\text { mm] }\end{array}$} & \multicolumn{3}{|c|}{$\begin{array}{c}\text { Сжатая поверхность, } \\
R \text { сжат }=21.0 \mathrm{~mm} \\
\text { [Compressed surface } \\
\left.R_{\text {com }}=21.0 \mathrm{~mm}\right]\end{array}$} & \multicolumn{3}{|c|}{$\begin{array}{c}\text { Ненагруженная (плоская) } \\
\text { поверхность } \\
\text { [Unloaded (flat) surface] }\end{array}$} \\
\hline & \multicolumn{2}{|c|}{ 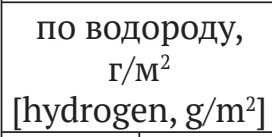 } & \multirow{2}{*}{$\begin{array}{c}\mathrm{KРПК} \\
\mathrm{Fe}^{2+}+\mathrm{Fe}^{3+} \\
\Gamma / \mathrm{M}^{2} \\
{[\mathrm{CDCP}} \\
\mathrm{Fe}^{2+} \mathrm{Fe}^{3+} \\
\left.\mathrm{g} / \mathrm{m}^{2}\right]\end{array}$} & \multicolumn{2}{|c|}{ 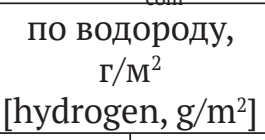 } & \multirow{2}{*}{$\begin{array}{c}\text { КРПК } \\
\mathrm{Fe}^{2+}+\mathrm{Fe}^{3+} \\
\Gamma / \mathrm{M}^{2} \\
{[\mathrm{CDCP}} \\
\mathrm{Fe}^{2+}+\mathrm{Fe}^{3+} \\
\left.\mathrm{g} / \mathrm{m}^{2}\right]\end{array}$} & \multicolumn{2}{|c|}{ 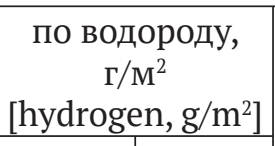 } & \multirow{2}{*}{$\begin{array}{c}\text { КРПК } \\
\mathrm{Fe}^{2+}+\mathrm{Fe}^{3+}, \\
\Gamma / \mathrm{M}^{2} \\
{[\mathrm{CDCP}} \\
\mathrm{Fe}^{2+}+\mathrm{Fe}^{3+}, \\
\left.\mathrm{g} / \mathrm{m}^{2}\right]\end{array}$} \\
\hline & $\begin{array}{l}\text { Всего } \\
\text { [total] }\end{array}$ & $\mathrm{Fe}^{2+}$ & & $\begin{array}{c}\text { Всего } \\
\text { [total] }\end{array}$ & $\mathrm{Fe}^{2+}$ & & $\begin{array}{l}\text { Всего } \\
\text { [total] }\end{array}$ & $\mathrm{Fe}^{2+}$ & \\
\hline 20 & 2.74 & 1.52 & 1.98 & 0.87 & 0.49 & 12.06 & 1. & 0.5 & 6.73 \\
\hline 40 & 13.70 & 7.62 & 4.61 & 3.17 & 1.77 & 13.82 & 4.03 & 2.31 & 8.98 \\
\hline 60 & 35.10 & 19.6 & 5.79 & 6.77 & 3.78 & 15.95 & 9.11 & 5.21 & 16.30 \\
\hline 80 & 68.41 & 38.32 & 12.38 & 11.59 & 6.48 & 19.74 & 16.25 & 9.29 & 19.70 \\
\hline 100 & 114.80 & 64.44 & 32.70 & 17.59 & 9.84 & 22.48 & 25 & 14. & 23.06 \\
\hline 120 & 175.25 & 98.56 & 56.29 & 24.73 & 13.83 & 26.69 & 36.71 & 21.00 & 30.06 \\
\hline 140 & 250.59 & 141.16 & 84.25 & 3.99 & 18.45 & 27.70 & 50.04 & 28.62 & 32.00 \\
\hline
\end{tabular}

о присутствии кислорода в реакционном пространстве.

На растянутой поверхности (см. табл.), кулонометрические и расчетно-водородные данные соответствуют друг другу только в начальный момент испытаний - через 20 минут. Далее количество железа, найденное методом КРПК, отстает от расчетного по водороду. Данное обстоятельство связано, вероятно, с развитием селективной коррозии, когда выход в среду продуктов коррозии компонентов тестируемого сплава: хрома, никеля и марганца превышает количество железа.

\section{ЗАКЛЮЧЕНИЕ}

Проведенные эксперименты показали, что кинетические закономерности коррозии сплава в условиях знакопеременной деформации представляют собой комплексную задачу. Необходимо не только учитывать специфику коррозионного поведения системы «поверхность сплава раствор» в условиях нагрузки, но и, что особенно важно, регистрировать коррозионные потери в малом временнум интервале экспозиции образца. Для более точного определения коррозионного процесса необходимо в дальнейшем проводить селективную регистрацию микроколичеств и других продуктов коррозии стали при их совместном присутствии в агрессивной среде.

С физической точки зрения при одновременном воздействии механического деформирова- ния и агрессивной среды в реакционном пространстве «твёрдое тело-электролит» возникают колебательно - и электронно-возбужденные состояния межатомных связей, стимулирующие коррозию компонентов сплава и, как следствие, формирование оксидного слоя продуктов взаимодействия металла со средой.

Нестабильный по структуре, упомянутый слой изменяет толщину в процессе формирования, что вносит неопределённость в результаты исследования коррозионной кинетики традиционными методами в начальный период коррозионного формирования и растворения пассивирующего слоя на более ранней стадии развития коррозии.

\section{ИСТОЧНИК ФИНАНСИРОВАНИЯ}

Работа выполнена при содействии ООО «ЛИНЭКО».

\section{КОНФЛИКТ ИНТЕРЕСОВ}

Авторы декларируют отсутствие явных и потенциальных конфликтов интересов, связанных с публикацией настоящей статьи.

\section{СПИСОК ЛИТЕРАТУРЫ}

1. Беренштейн Г. В., Дьяченко А. М., Русанов А. И. Механохимический эффект растворения // Докл. АН СССР, 1988, т. 298(6), с. 1402-1404.

2. Rusanov A.I., Uriev N.B., Eryukin P.V., Movchan T. G., Esipova N.E. Effect of the strain sign in corrosion under stress // Mendeleev Commun., 2004, 
v. $14(2)$, pp. $58-59$. DOI: $10.1070 / \mathrm{mc} 2004 \mathrm{v} 014 \mathrm{n}$ 02abeh001875

3. Русанов А. И., Урьев Н. Б., Ерюкин П. В., Мовчан Т. Г., Есипова Н. Е. Открытие эффекта знака деформации в явлениях коррозии под напряжением // ДАН, 2004, т. 395(3), с. 364-366.

4. Есипова Н. Е., Блинов Е. Б., Мовчан Т. Г., Банных И. О. Исследование коррозионной стойкости изогнутой пластины из высокоазотистой немагнитной стали 05Х22АГ15Н8М2Ф в агрессивных средах // Металлы, 2007(2), с. 69-75.

5. Мовчан Т. Г., Есипова Н. Е., Ерюкин П.В., Урьев Н. Б., Русанов А. И. Механохимические эффекты в процессах коррозии металлов // Журнал общей химии, 2005, т. 75(11, с. 1761-1766.
6. Русанов А. И. Термодинамические основы механохимии. СПб.: Наука, 2006, 221 с.

7. ГОСТ 9.908-85. Металлы и сплавы. Межгосударственный стандарт.

8. Кузмак А. Е., Кожеуров А. В., Ефименко Л. А., Илюхин В.И. Кулонометрическая оценка коррозии околошовной зоны сварного шва при деформационном старении // Коррозия: материалы и защита, 2009(1), c. 43-46.

9. Кузмак А. Е., Кожеуров А. В. Кулонометрическая оценка скорости коррозии углеродистой стали // Защита Металлов, 2004, т. 40(3), с. 315-320.

10. Кузмак А. Е., Кожеуров А. В., Марин А. В. Кулонометрическая оценка влияния технологии сварки стали 12X18Н10Т на коррозию сварных зон // Коррозия: материалы и защита, 2005(1), с. 43-46.

\title{
EXPERIENCE OF THE CULONOMETRIC ESTIMATION OF THE CORROSION-ACTIVE SURFACE OF AUSTENITIC STEEL IN AN AGGRESSIVE ELECTROLYTE AT CONVERTIBLE DEFORMATION
}

\author{
(C2019 A. E. Kuzmak $\bowtie$, N. E. Esipova, A. V. Kozheurov \\ Frumkin Institute of Physical Chemistry and Electrochemistry, Russian Academy of Sciences (IPCE RAS) \\ 31, Leninsky pr., 119071 Moscow, Russian Federation
}

\begin{abstract}
Purpose.The paper investigates the electrochemical behaviour of a complex alloy 05H22AG15N8M2F-Sh (iron $55 \%$, chromium $20 \%$, nickel $8 \%$, manganese $15 \%$, nitrogen $0.5 \%$, and sulphur $<0.005$ ) in a $3 \mathrm{M} \mathrm{HCl}$ solution with static deformation of the variable sign by precision registration of corrosion products in the microgram range of corrosion loss.

Methods and Methodology.As shown in the present paper, Coulometric Registration of Corrosion Products (CDCP method) is an efficient approach to solving the problem of quantitative registration of a trace quantity of a substance for systems with different structural-phase surface conditions. The capabilities of the CDCP method were compared with the traditional (bulk) method to develop a methodology for assessing the effect of the strain sign on the corrosion kinetics of a multiphase alloy.

Results. The CDCP method was used to obtain the kinetic dependences of the accumulation of the ions: $\mathrm{Fe}^{2+}$ and $\mathrm{Fe}^{3+}$ corrosion products in the medium and on a compressed and stretched surfaces at different radii of curvature under the conditions of stationary stress.

It is shown that the use of the CDCP method is a promising way of significantly expanding the possibilities of corrosion studies of complex corrosion systems.

Conclusions. The advantages of the CDCP method for studying the kinetic regularities of a complex alloy corrosion under alternating deformation conditions were achieved. The possibility of conducting selective registration of a trace quantity of stainless steel corrosion products with their combined presence in an aggressive environment was shown.

The role of the oxide layer of the products of the metal interaction with the medium were established.
\end{abstract}

$\triangle$ Kuzmak Alexander Evseevich, e-mail: akuzmak@yandex.ru 
According to the results, it can be concluded that it is necessary to study in detail the kinetics of the formation and dissolution of the passivating layer, and, consequently, the feasibility of conducting corrosion tests at an early stage of the corrosion process.

Keywords: corrosion, deformation, coulometry, volumetry, surface stress.

\section{SOURCE FINANCING}

LINEKO Scientific Production Company.

\section{CONFLICT OF INTEREST}

The authors declare the absence of obvious and potential conflicts of interest related to the publication of this article.

\section{REFERENCES}

1. Berenshtein G. V., Dyachenko A. M., Rusanov A. I. Mekhanohimicheskij effekt rastvoreniya [Mechanochemical effect of dissolution] Report Academy of Sciences of the USSR, 1988, v. 298 (6), pp. 1402-1404. (in Russ.)

2. Rusanov A. I., Uriev N. B., Eryukin P. V., Movchan T. G., Esipova N.E. Effect of the strain sign in corrosion under stress. Mendeleev Commun., 2004, v. 14(2), pp. 58-59.DOI: 10.1070/mc2004v014n02abeh 001875

3. Rusanov A. I., Ur'ev N. B., Eryukin P. V., Movchan T. G., Esipova N. E. Otkrytie effekta znaka deformacii v yavleniyah korrozii pod napryazheniem [Discovery of the sign deformation effect in stress corrosion phenomena]. Report Academy of Sciences, 2004, v. 395(3), pp. 364-366. (in Russ.)

4. Esipova N. E., Blinov E. B., Movchan T. G., Bannykh I. O. Corrosion Resistance of a Bent Plate from a High-Nitrogen Nonmagnetic 05Kh22AG15N8M2F
Steel in Aggressive Media. Russian metallurgy (Metally), 2007(2), pp. 148-75.

5. Movchan T. G., Esipova N. E., Eryukin P. V., Uryev N. B., Rusanov A. I. Mechanochemical effects in processes of corrosion of metals. Russian Journal of General Chemistry, 2005, v. 75(11), pp. 1681-1686. DOI: 10.1007/s11176-005-0491-8

6. Rusanov A. I. Termodinamicheskie osnovy mekhanohimii [Thermodynamic principles of mechanochemistry]. Saint Petersburg, Nauka Publ., 2006, 221 p. (in Russ.)

7. GOST 9.908-85. Metals and alloys. Interstate standard. (in Russ.)

8. Kuzmak A. E., Kozheurov A. V., Efimenko L. A., Ilyukhin V. I. Kulonometricheskaya ocenka korrozii okoloshovnoj zony svarnogo shva pri deformacionnom starenii [Coulometric corrosion assessment of the heat-affected weld zone during strain aging]. Korroziya: Materialy, Zashchita, 2009(1), pp. 43-46. (in Russ.)

9. Kuzmak A. E., Kozheurov A. V. Kulonometricheskaya ocenka skorosti korrozii uglerodistoj stali [Coulometric corrosion rate assessment for carbon steel]. $\mathrm{Za}$ shchita Metallov, 2004, v. 40(3), pp. 315-320. (in Russ.)

10. Kuzmak A. E., Kozheurov A. V., Marin A. V. Coulometric evaluation of influence of the welding technology of 12Kh18N10T steel on welded zone corrosion. Korroziya: Materialy, Zashchita, 2005(1), pp. 4346. (in Russ.)
Кузмак Александр Евсеевич - к. ф.-м. н., зав. сектором, Институт физической химии и электрохимии им. А. Н. Фрумкина РАН, Москва, Российская Федерация; e-mail: akuzmak@yandex.ru. Scopus Author ID: 6603371209.

Eсипова Н. E. - к. х. н., с. н. с., Институт физической химии и электрохимии им. А.Н. Фрумкина РАН, Москва, Российская Федерация; e-mail: esipova@phyche.ac.ru

Кожеуров А. В. - м. н. с., Институт физической химии и электрохимии им. А. Н. Фрумкина РАН, Москва, Российская Федерация.
Kuzmak Alexander E. - Cand. Sci. (Phys.-Math), Head of Sector, Frumkin Institute of Physical Chemistry and Electrochemistry, Russian Academy of Sciences, Moscow, Russian Federation; e-mail: akuzmak@yandex.ru. Scopus Author ID: 6603371209.

Esipova N. E. - Cand. Sci. (Chem.), Senior Researcher, Frumkin Institute of Physical Chemistry and Electrochemistry, Russian Academy of Sciences, Moscow, Russian Federation; e-mail: esipova@phyche.ac.ru.

Kozheurov A. V. - Junior Researcher, Frumkin Institute of Physical Chemistry and Electrochemistry, Russian Academy of Sciences, Moscow, Russian Federation. 